\title{
Decaying warm dark matter and structure formation
}

\author{
Jui-Lin Kuo ${ }^{a}$ Massimiliano Lattanzi ${ }^{b}$ Kingman Cheung ${ }^{a, c, d}$ José \\ W. F. Valle
}

\author{
${ }^{a}$ Department of Physics, National Tsing Hua University, Hsinchu, Taiwan, \\ ${ }^{b}$ Istituto Nazionale di Fisica Nucleare, sezione di Ferrara, Polo Scientifico e Tecnologico, \\ Edificio C, Via Saragat 1, I-44122 Ferrara, Italy \\ ${ }^{c}$ Physics Division, National Center for Theoretical Sciences, Hsinchu, Taiwan, \\ ${ }^{d}$ Division of Quantum Phases and Devices, School of Physics, Konkuk University, Seoul \\ 143-701, Republic of Korea \\ ${ }^{e}$ AHEP Group, Instituto de Física Corpuscular, C.S.I.C./Universitat de Valéncia, Campus \\ de Paterna, Apartado 22085, E-46071 Valéncia, Spain \\ E-mail: juilinkuo@gapp.nthu.edu.tw, lattanzi@fe.infn.it, cheung@phys.nthu.edu.tw, \\ valle@ific.uv.es
}

\begin{abstract}
.
We examine the cosmology of warm dark matter (WDM), both stable and decaying, from the point of view of structure formation. We compare the matter power spectrum associated to WDM masses of $1.5 \mathrm{keV}$ and $0.158 \mathrm{keV}$, with that expected for the stable cold dark matter $\Lambda \mathrm{CDM} \equiv \mathrm{SCDM}$ paradigm, taken as our reference model. We scrutinize the effects associated to the warm nature of dark matter, as well as the fact that it decays. The decaying warm dark matter (DWDM) scenario is well-motivated, emerging in a broad class of particle physics theories where neutrino masses arise from the spontaneous breaking of a continuous global lepton number symmetry. The majoron arises as a Nambu-Goldstone boson, and picks up a mass from gravitational effects, that explicitly violate global symmetries. The majoron necessarily decays to neutrinos, with an amplitude proportional to their tiny mass, which typically gives it cosmologically long lifetimes. Using N-body simulations we show that our DWDM picture leads to a viable alternative to the $\Lambda \mathrm{CDM}$ scenario, with predictions that can differ substantially on small scales.
\end{abstract}




\section{Contents}

1 Introduction 1

2 The simulations $\quad 4$

2.1 Methodology 4

$\begin{array}{lll}2.2 & \text { Initial Conditions } & 7\end{array}$

2.3 Numerical convergence tests 8

3 Simulation Results $\quad 11$

3.1 Density Field 12

3.2 Matter Power Spectrum 14

3.3 Halo Mass Function 18

3.4 Effects of baryonic physics 20

4 Conclusions $\quad 21$

A Results for $5.3 \mathrm{keV}$ decaying dark matter 23

\section{Introduction}

So far we have failed to identify the nature of what makes up most of the matter present in the Universe, only a small fraction of which is the baryonic stuff found in stellar objects and intergalactic medium. The existence of a "dark matter" component on all scales is inferred mainly from the gravitational effect it seems to have on visible matter. No particle of the standard model can play the role of dark matter, hence it must be new physics. For several decades already, there seems to be a consensus that dark matter must be collisionless yet, to date, its detailed nature remains a mystery [1].

On the other hand, the discovery of neutrino oscillations $[2,3]$ indicates the need for nonzero neutrino masses. However, underpinning the detailed properties of neutrinos and the ultimate origin of their mass poses another great challenge for the standard model of particle physics [4]. 
A tantalizing possibility is that cosmological dark matter is deeply related to the generation of neutrino masses [5]. For example, dark matter could be a messenger particle associated to the neutrino mass generation [6-9]. Its stability could also reflect a fundamental property of neutrinos, such as its possible Dirac nature [10]. Or it could follow, for example, as a remnant of the symmetry which accounts for the peculiar pattern of neutrino mixing angles indicated by the oscillation experiments $[11,12]$. In both cases dark matter would be a stable weakly interacting massive particle (WIMP). There are, however, many well-motivated alternatives to WIMP dark matter. The associated dark matter candidates need not be strictly stable, while providing viable cosmology. For example, a decaying gravitino [13, 14] provides an attractive scenario for decaying dark matter related to neutrino physics.

Here we focus on the possibility that the majoron $J$ plays the role of decaying dark matter. This has a two-fold motivation. Theoretically, the majoron is a very broad concept, emerging as a Nambu-Goldstone boson in any theory where neutrino masses arise from the spontaneous breaking of a continuous global symmetry, such as lepton number $[15,16]$. On the other hand, as an alternative to the $\Lambda \mathrm{CDM}$ paradigm, the majoron picture may have the right properties to address some potential drawbacks of the standard scenario, such as the "small scale crisis" which can be alleviated by the warm nature of the majoron $[17,18]$.

The majoron is assumed to acquire a mass $m_{J}$ through gravitational instanton effects that explicitly violate global symmetries [19]. The value $m_{J}$ of the majoron mass can not be computed by theory. A particularly interesting range for the mass is the $\mathrm{keV}$ range. Such keV majoron has been suggested by Berezinsky and Valle (BV) as a viable decaying dark matter candidate [20]. On general theoretical grounds, the massive majoron is necessarily unstable, as it couples to neutrinos, with a strength proportional to their tiny mass [16]. In order for the majoron to be the dark matter, it must be cosmologically long-lived, i.e. its lifetime $\tau_{J}$ should be of the order of the age of the Universe $t_{0}=13.8 \mathrm{Gyr} \simeq 4 \times 10^{17} \mathrm{~s}$, or larger, $\tau_{J} \gtrsim t_{0}$. In fact, it has been shown that cosmic microwave background (CMB) data places a stronger requirement on the majoron decay rate [21], in order to avoid producing too much fluctuation power on the largest CMB scales, following the decay of majoron to neutrinos and the subsequent modifications to the cosmological gravitational potentials. In the framework of a simple one-parameter extension of the standard $\Lambda \mathrm{CDM}$ model, one finds $\tau_{J}>50 \mathrm{Gyr}$ using WMAP9 data [22]. The limit tightens to $\tau_{J}>160$ Gyr when Planck 2013 data and large-scale structures linear data from WiggleZ and BOSS are taken into account ${ }^{1}$ [23]. Here we adopt the most conservative limit as our reference choice when discussing DWDM.

Indeed, the massive majoron dark matter model has been shown to be consistent with

\footnotetext{
${ }^{1}$ Note however, that this last limit is obtained assuming a model with primordial tensor modes, motivated at the time by the BICEP2 claim, so the two limits cannot be directly compared.
} 
CMB data for interesting choices of the relevant parameters [21-23]. If the majoron was in thermal equilibrium with the plasma in the early Universe and decoupled at some later stage, a mass $m_{J}=\mathcal{O}(\mathrm{keV})$ would produce the right dark matter abundance [20]. Moreover, a thermal particle with $\mathrm{keV}$ mass would be a WDM candidate. For a thermal majoron that decoupled when all the degrees of freedom of the standard model were still excited, measurements of CMB anisotropies yield the following constraints [22]: $m_{J}=(0.158 \pm 0.007) \mathrm{keV}$ (68\% C.L.) and $\tau_{J}>50 \mathrm{Gyr}$ (95\% C.L). We note that this value of the mass is in tension with constraints coming from observations of the Ly- $\alpha$ forest [24-26]. There is the alternative possibility that the majoron was never in thermal equilibrium with the other species in the cosmological plasma. For this reason ${ }^{2}$, we will treat the mass of the majoron as a free parameter and consider different values for it in our study.

Since the coupling of the majoron to neutrinos $g_{\nu}$ is proportional to the neutrino mass [16], the decay $J \rightarrow \nu \nu$ can naturally have a very long lifetime on cosmological scales. The CMB constraints for the thermal majoron can be shown to imply $g_{\nu}<5 \times 10^{-18}$. Other decay channels may be present, depending on the model. For example, in type II seesaw models the majoron can also decay to photons. The effective (one-loop suppressed) coupling to $\gamma$ 's can be constrained through X-and $\gamma$-ray observations $[22,27]$ However, since this coupling is rather model dependent, we will disregard the radiative decay channel in what follows. This is, in practice, equivalent to assume that neutrino masses are generated through simplest type I seesaw mechanism.

In the present paper we examine the effect of decaying warm dark matter on non-linear structure formation, so far unexplored in the literature. The aim of this paper is exactly to fill this gap, and study the effect of decaying majoron dark matter on structure formation using N-body simulations. In fact, we show that such a DWDM majoron expected within the BV framework does indeed yield a viable cosmology, which can differ substantially from that of the standard $\Lambda \mathrm{CDM}$ paradigm. This happens for two reasons. First, due to the warm nature of the majoron and second, due to the fact that it decays. Our paper is organized as follows. In section 2 we explain the approach employed in our N-body simulations and demonstrate the convergence of our methodology. In section 3 we describe our results while in section 3.4 we discuss about the possible impact of various baryonic processes. Finally, in section 4 we draw our conclusions and summarize our results, commenting on their possible implications. Additional discussion on structure formation and the WDM mass allowed by Lyman-alpha forest data is given in the appendix.

\footnotetext{
${ }^{2}$ In fact, there are no model-independent limits on the majoron mass.
} 


\begin{tabular}{c|c|c|c}
\hline \hline Abbreviations & Initial Conditions & Lifetime & WDM mass \\
\hline SCDM & CDM & $\infty$ & N $/ A$ \\
DCDM & CDM & $50 \mathrm{Gyr}$ & N $/ A$ \\
SWDM-M & WDM & $\infty$ & $1.5 \mathrm{keV}$ \\
DWDM-M & WDM & $50 \mathrm{Gyr}$ & $1.5 \mathrm{keV}$ \\
SWDM-m & WDM & $\infty$ & $0.158 \mathrm{keV}$ \\
DWDM-m & WDM & $50 \mathrm{Gyr}$ & $0.158 \mathrm{keV}$ \\
\hline \hline
\end{tabular}

Table 1. The abbreviations and features of the simulations we have performed in this article. To avoid word cluttering in the following we will use these abbreviations.

\section{The simulations}

\subsection{Methodology}

In order to scrutinize the novel features of the DWDM scenario we perform different cosmological simulations, as listed in Table. 1. We consider DM that is either stable or that decays with a lifetime of $50 \mathrm{Gyr}$, which is the lower limit from the CMB obtained in Ref. [22]. We also consider the case of CDM, and two different cases of WDM. This makes a total of six N-body cosmological simulations. To avoid word cluttering in the following, we use abbreviations for these simulations, as given in the Tab. 1.

In the CDM simulations, the mass of the DM particle is large enough to suppress freestreaming on the initial matter power spectrum. In other words, this is the limit of the DM temperature-to-mass ratio going to zero. In the DWDM case, we consider two values of the DM mass, namely $m_{J}=0.158 \mathrm{keV}$ and $m_{J}=1.5 \mathrm{keV}$. The former value, as mentioned in Sec. 1 , is the one that would give the right relic density for a scalar particle, like the majoron, that decoupled in the early Universe when all the degrees of freedom of the standard model were present. The latter value can be realized if the majoron has a nonthermal distribution, or if it is thermal but its density is diluted by an additional production of entropy after decoupling (both possibilities were described by an effective parameter called $\beta$ in [21]). In any case, we will remain agnostic about the production mechanism, and assume a thermal distribution in all our WDM simulations when generating initial conditions (see below). This is also in view of the fact that even if the majoron provides a neat particle physics motivation for the DWDM scenario, nevertheless our results are more general, in the sense that they apply independently of the particular nature of DM.

The values that we choose for the DM mass are in tension with lower limits obtained 
from observations of Ly- $\alpha$ flux-power spectra. For example, the recent analysis of Ref [26] finds $m>5.3 \mathrm{keV}$ at $95 \% \mathrm{CL}$ for a thermal candidate, from a combined analysis of the XQ100 and HIRES/MIKE data samples. This limit can be relaxed to $3.5 \mathrm{keV}$ by allowing for a non-smooth evolution of the temperature of the intergalactic medium (IGM). We choose to consider smaller values of the mass for two reasons. The first one is that the nature of our paper is exploratory, and the main purpose is to study the joint effects of the DM decay and free streaming. A small value of the mass allows us to maximize free-streaming in order to better highlight the interplay between these two effects, taking into account also the computational resources at our disposal. The second reason is that the interpretation of Ly $-\alpha$ data is somehow complicated by several factors, like for example the aforementioned dependence on the modeling of the IGM thermal history. For example, Ref [28] finds that the Ly $-\alpha$ data can be made consistent with models excluded by other analyses. This, however, does not necessarily imply that thermal DM with the masses considered here can be made consistent with Ly $-\alpha$ observations; a dedicated study would be necessary for that purpose. That said we have, in any case, also performed simulations for "large" DM mass, $m_{J}=5.3 \mathrm{keV}$. We found no appreciable difference with the CDM case in the range of scales that we are able to probe within our numerical resolution. The results for that case are given in the appendix. A future analysis might consider different values of the mass, using larger-resolution simulations, and also a non-thermal spectrum for the DM.

The standard N-body simulation code Gadget2 [29] is adopted to perform the simulations. Gadget2 follows the evolution of a self-gravitating system of collisionless "particles", taking into account the expansion of the Universe. These particles are in fact macroscopical objects, composed by a large number of DM particles. For this reason one usually refers to them as "simulation particles", as opposed to actual DM particles. In order to implement the effect of decay, we include two modifications in the original Gadget2 code, following the approach in Refs. [30, 31], which addressed the issue of dark matter decays into dark radiation. Although here we are concerned with dark matter decaying into relativistic neutrinos, the algorithm of the simulation is similar to Ref. [31]. First of all, the mass of the simulation particle is reduced by a small amount at each step in the simulation, in order to account for the effect of DM decay. Therefore, in the simulation the mass of the simulation particles is altered according to

$$
M(t)=M\left(1-R+R e^{-t(z) / \tau_{J}}\right),
$$

where $M$ is the initial mass of the simulation particles, and $R \equiv\left(\Omega_{M}-\Omega_{b}\right) / \Omega_{M}$ is the DM fraction in the matter component, and $\Omega_{b}$ refers to the baryon contribution. In addition to reducing the simulation particle mass, we also modify the expansion rate of the universe in accordance with the energy content at each redshift. Due to the dark matter decaying 
into relativistic particles (in the case of the majoron, neutrinos), the expansion history in the DWDM majoron scenario is different from that of the stable DM case. The evolution of the dark matter and of the decay products $\rho_{d m}$ and $\rho_{d p}$ are described by

$$
\begin{aligned}
\dot{\rho}_{d m}+3 \mathcal{H} \rho_{d m} & =-\frac{a}{\tau_{J}} \rho_{d m}, \\
\dot{\rho}_{d p}+4 \mathcal{H} \rho_{d p} & =\frac{a}{\tau_{J}} \rho_{d m},
\end{aligned}
$$

where $\mathcal{H}$ and $a$ are the conformal Hubble parameter and the scale factor, and the dot represents the derivative with respect to the conformal time. Here we assume that the decay products are relativistic, so the pre-factor for the Hubble drag term for this component in Eq. (2.2) is 4 . On the other hand, $\mathcal{H}$ at each redshift is determined by

$$
\mathcal{H}^{2}(z)=\frac{8 \pi G}{3} a^{2}\left(\rho_{d m}(z)+\rho_{b}(z)+\rho_{d p}(z)+\rho_{\Lambda}(z)\right),
$$

where $G, \rho_{b}$, and $\rho_{\Lambda}$ are the gravitational constant, the baryon energy density, and the energy density of dark energy, respectively. We assume that dark energy is in the form of a cosmological constant. We also neglect the presence of the thermal relic neutrinos produced in the early phases of the cosmological evolution, both at the background and perturbation level. Note that $\rho_{b}$ and $\rho_{\Lambda}$ are unaffected by the energy exchange between DM and the decay products, hence they evolve as in the standard case (i.e., $\rho_{b} \propto a^{-3}$ and $\rho_{\Lambda}=$ const). Therefore, given the initial values for $\rho_{d m}$ and $\rho_{d p}$, we need to numerically solve Eq. (2.2) in conjunction with Eq. (2.3) at each timestep, in order to obtain the precise Hubble parameter describing the expansion of the universe.

For simplicity, in the simulation we neglect the effects of perturbations in the decay products. Indeed, we note that the contribution of the decay products to the energy density is very small, since we consider very long DM lifetimes. Moreover, the decay-produced neutrinos are free-streaming and thus do not cluster, due to their relativistic nature. So the main effect of the decay products is just to reduce the amount of matter that is able to cluster, and this is fully captured by decreasing the mass of each simulation particle as in Eq. 2.1. We expect this approximation to break down on the largest scales, above the free-streaming length of the decay products, where these are able to cluster. However, this happens around the horizon scale, which is much larger than the largest scales probed by our simulations, that use a box size of $50 h^{-1} \mathrm{Mpc}$. Moreover, the power spectrum on those scales can be reliably computed using linear theory, if necessary. As a result, we expect that addional effects related to perturbations in the decay-produced neutrinos will be subtle and not change our results significantly.

Note that we do not include baryons in our simulation and thus neglect, among others, baryonic feedback processes. The reason again is that, given the scope of our paper, we 

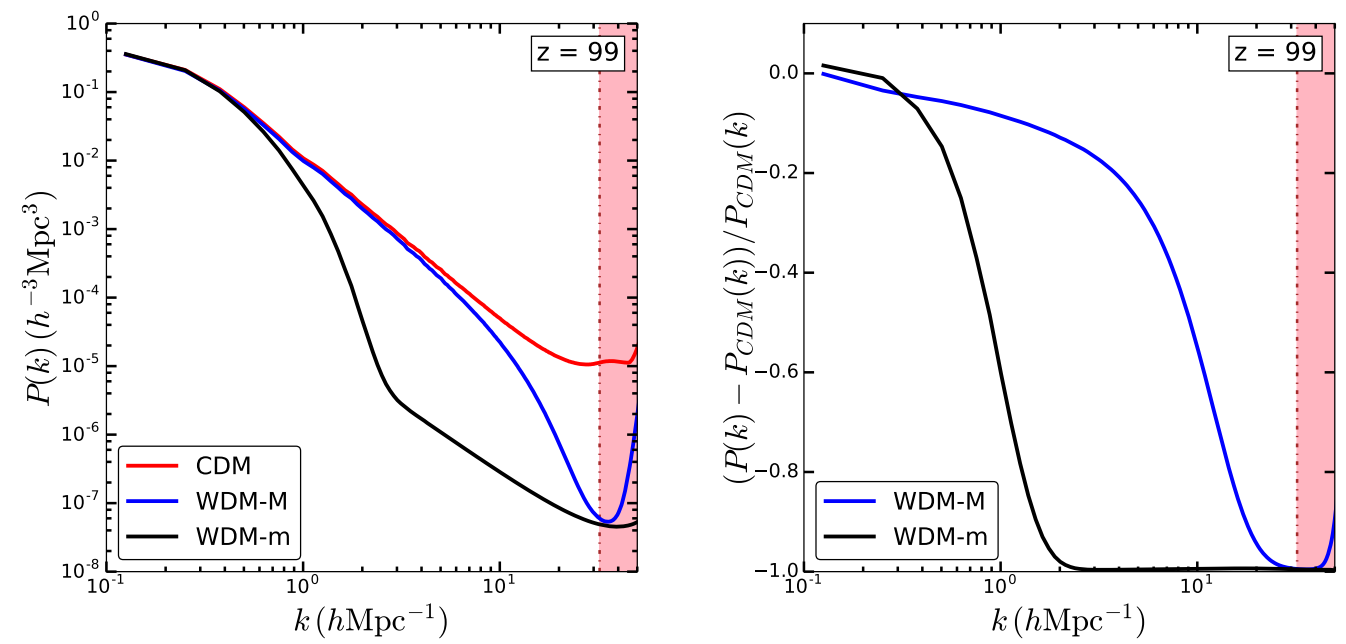

Figure 1. Left panel: comparison of the matter power spectrum of initial condition for $\Lambda$ CDM (red solid line), WDM with mass $1.5 \mathrm{keV}$ (blue solid line) and WDM with mass $0.158 \mathrm{keV}$ (black solid line). The vertical "Nyquist" band lies above the limit set by the scale of the average size of the simulation particle. Right panel: Relative difference between the WDM and CDM power spectra, for WDM with mass $1.5 \mathrm{keV}$ (blue solid line) and $0.158 \mathrm{keV}$ (black solid line). Here the matter power spectra are obtained from the output of 2LPTic, hence the effect of finite numerical resolution is already included. The cut-off due to the free-streaming of WDM can be clearly seen.

want to focus on the interplay between DM decay and free streaming. The inclusion of baryonic effects, through hydrodynamic simulations, would be of course mandatory for a rigorous comparison between the predictions of the "full-fledged" DWDM scenario and the observations. We comment, anyway, on the possible effects of baryonic physics in Sec. 3.4.

\subsection{Initial Conditions}

To generate initial conditions for the N-body simulations, one uses linear theory to evolve the primordial perturbations in $k$ space up to some redshift deep in the matter-dominated era, but still early enough for the linear predictions to be valid. This is the initial redshift, in our case $z=99$, from which the N-body simulations start. Since this initial time is well before the DM decay kicks off, the initial power spectra for the stable and decaying DM case are the same. We adopted the fitting form of CDM matter power spectrum $P_{\mathrm{CDM}}$ given in Ref. [32], which is based on the calculation of linear theory, to compute the initial power spectrum for CDM initial conditions. In the WDM scenario, we estimate the power spectrum at the initial redshift as

$$
P_{\mathrm{WDM}}(k)=T_{\mathrm{WDM}}^{2}(k) \times P_{\mathrm{CDM}}(k),
$$


where $T_{\mathrm{WDM}}(k)$ is the transfer function given in Ref. [33] (where it is called $T_{\chi}$ ), which accounts for the cut-off in the matter power spectrum due to the free-streaming effect. The initial transfer function for thermal WDM can be written as

$$
T_{\mathrm{WDM}}(k)=\left(1+(\alpha k)^{2 \nu}\right)^{-5 / \nu},
$$

where $\alpha=0.048\left(\Omega_{D M} / 0.4\right)^{0.15}(h / 0.65)^{1.3}\left(\mathrm{keV} / m_{\mathrm{DM}}\right)^{1.15}(1.5 / g)^{0.29} \mathrm{Mpc}$ and $\nu=1.2$. Here $\Omega_{D M}$ is the dark matter energy density, $m_{\mathrm{DM}} \equiv m_{J}$ is the dark matter mass and $g$ is the effective number of dark matter degrees of freedom ( $g=1$ for the majoron). Note that $\alpha$ is a critical length that determines the cut-off scale in the initial power spectrum. Using $m_{J}=0.158 \mathrm{keV}$ or $1.5 \mathrm{keV}, g=1$, and the values listed below for the other parameters, one has that the transfer function reduces the initial fluctuation power to a fraction $1 / e$ of the corresponding $\mathrm{CDM}$ value at $k \simeq 1$ and $17 h \mathrm{Mpc}^{-1}$, respectively. We can take these values as rough estimates of the free-streaming wavenumber.

In order to generate the initial condition for the cosmological simulation we used the 2LPTic code [34], based on the second-order Lagrangian perturbation theory. In Fig. 1, we show the initial $(z=99)$ power spectra for CDM and for the two WDM models considered here, given by 2LPTic, hence numerical limitations are already included ${ }^{3}$.

Note that when we extract initial conditions from the power spectrum, we use the same random seed for each pair of stable/decaying DM simulations. In other words, the two simulations of each pair have exactly the same initial conditions. The simulations start from redshift $z=99$. The input cosmological parameters are: the matter energy density $\Omega_{m}=0.3$, the cosmological constant energy density $\Omega_{\Lambda}=0.7$, the baryon energy density $\Omega_{b}=0.04$, the dimensionless Hubble constant $h=0.7$, the scalar spectral index $n_{s}=0.96$, and the power spectrum normalization factor $\sigma_{8}=0.8$. For WDM simulations, we input thermal velocities at $z=99$ to the simulation particles, consistently with the initial spectrum. This has however a negligible effect on nonlinear structure formation since thermal velocities have already decayed out at $z=99$, due to the expansion of the Universe. We have used $512^{3}$ simulation particles and a cube containing these particles with each side equals to $50 h^{-1} \mathrm{Mpc}$. The mass $M_{\text {sim }}$ of each simulation particle at the initial time is $M_{\text {sim }} \simeq 7.8 \times 10^{7} h^{-1} M_{\odot}$. Periodic boundary conditions are employed in order to avoid boundary effects.

\section{$2.3 \quad$ Numerical convergence tests}

In this section we quantify the degree of convergence of our simulations. We do this by considering simulations with different volume and number of particles. In particular, we

\footnotetext{
${ }^{3}$ The sudden change of slope for the WDM-m scenario around $k=3 h \mathrm{Mpc}^{-1}$ is due to the presence of shot noise, which will be discussed in Sec. 2.3.
} 
change the resolution of the simulations at fixed volume, or change the size of the simulation volume at fixed resolution. This will allow us to assess numerical limitations and to define the limit of validity of the results inferred from our simulations.

There are two kinds of numerical limitations. The first is sample variance, also known as "cosmic variance". In the simulation, the source of sample variance is the finite volume of the simulation and the fact that each simulation only provides a single realization of the underlying statistical distribution of particles. The sample variance prevents us from precisely predicting the density field on large scales [35]. We use for our simulations a box size of $50 h^{-1} \mathrm{Mpc}$, corresponding to a fundamental mode $k \simeq 0.13 \mathrm{~h} \mathrm{Mpc}^{-1}$. The second kind of numerical limitation is due to the discreteness of the simulation particles, i.e. to the fact that we adopt particles to represent a continuous density field. The overall resolution limit of the simulations is set by both the box size and the number of particles, and is described by the Nyquist wavenumber $k_{\mathrm{Nyq}}$

$$
k_{\mathrm{Nyq}}=\pi(N / V)^{1 / 3} .
$$

Beyond the Nyquist wavenumber, the accuracy of the power spectrum is strongly degraded. For the parameters used in our baseline simulations, $k_{\mathrm{Nyq}} \simeq 32 \mathrm{~h} \mathrm{Mpc}^{-1}$.

The finite resolution of the simulations has two consequences. First, non-zero power exists on all scales, called shot noise. The shot noise originates because we adopt particles to represent a continuous density field. The amplitude of the shot noise is independent of the wavenumber $k$ and depends instead on the number of particles in the simulation, and therefore on the resolution $[35,36]$. The second consequence is a discreteness peak in the power spectrum at twice the Nyquist limit. This small excess of power is a common feature of all the N-body simulations. This is however more of a problem for WDM simulations than $\mathrm{CDM}$ ones, since the former have much less power at small scales, making this numerical artifact more manifest. This causes the well-known spurious halo issues in standard WDM simulations [37]. We will discuss this effect in more details in Sec. 3.3.

As anticipated above, in order to test the convergence of our simulations, we compare the results from runs with different box size and particle resolution. To this purpose, we perform simulations with $N=128^{3}, 256^{3}, 512^{3}$ and $L=V^{1 / 3}=50,100 h^{-1} \mathrm{Mpc}$. We then compare the resulting power spectra at $z=0$ with that from our baseline run with $L=50 h^{-1} \mathrm{Mpc}$ and $N=512^{3}$. We concentrate on the DWDM-m case, i.e. the one with the smaller mass for the dark matter particle. This is because this is the case with the stronger suppression of small-scale power, for which numerical issues at small scales are thus in principle more relevant. In order to assess the impact of box size, we do the following. We compute the ratio of the matter power spectra at $z=0$ from the simulations with 


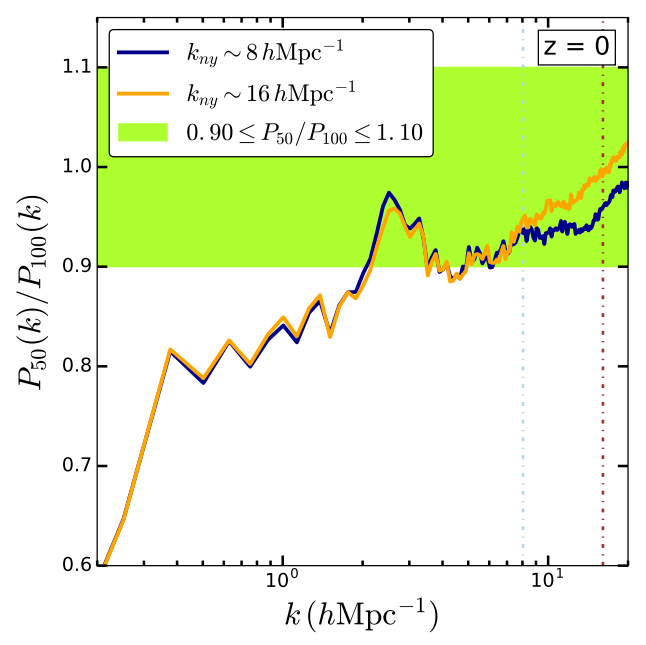

Figure 2. Ratio of power spectra obtained from simulations with box-size $L=50$ and $100 h^{-1} \mathrm{Mpc}$, at fixed resolution. The blue and yellow curves correspond to $k_{\mathrm{ny}} \simeq 8$ and $16 h \mathrm{Mpc}^{-1}$, respectively. The green band shows a $10 \%$ deviation between the spectra.

$\{L, N\}=\left\{50 h^{-1} \mathrm{Mpc}, 128^{3}\right\}$ and $\left\{100 h^{-1} \mathrm{Mpc}, 256^{3}\right\}$. The latter has different number of

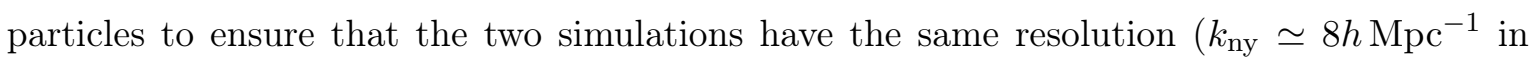
both cases) and that we are isolating the effects of the finite simulation volume. The ratio between the spectra should give us a rough measure of the numerical error associated to a finite volume size of $50 h^{-1} \mathrm{Mpc}$, at that resolution. We also do the same for the pair of simulations with $\{L, N\}=\left\{50 h^{-1} \mathrm{Mpc}, 256^{3}\right\}$ and $\left\{100 h^{-1} \mathrm{Mpc}, 512^{3}\right\}\left(k_{\mathrm{ny}} \simeq 16 h \mathrm{Mpc}^{-1}\right)$ to be confident that our results reliably extrapolate to our reference simulation with $\{L, N\}=$ $\left\{50 h^{-1} \mathrm{Mpc}, 512^{3}\right\}$ and $k_{\mathrm{ny}} \simeq 32 h \mathrm{Mpc}^{-1}$. Of course a more direct way would be to perform a simulation with $\{L, N\}=\left\{100 h^{-1} \mathrm{Mpc}, 1024^{3}\right\}$, but we choose not to follow this path due to our limited computational resources.

We show the ratio of the spectra computed in this way in Fig. 2. It is evident how the large-scale power of the simulations does not match due to the cosmic variance. However, we see that for both resolutions, the relative difference between $L=50$ and $100 h^{-1} \mathrm{Mpc}$ is $10 \%$ or better at wavenumbers above $k \simeq 2 h \mathrm{Mpc}^{-1}$. This makes us confident that the same applies at the resolution of our reference simulation.

Then, to study the effect of the finite resolution, in the left panel of Fig. 3 we show matter power spectra at $z=0$ from simulations with $50^{3} h^{-3} \mathrm{Mpc}^{3}$ box size and $N^{1 / 3}=$ $128,256,512$. Values of $k_{\mathrm{Ny}}$ for this runs are 8, 16 and $32 \mathrm{~h} \mathrm{Mpc}^{-1}$. The right panel of the same figure shows the corresponding plot for a $100^{3} h^{-3} \mathrm{Mpc}^{3}$ box size, with $k_{\mathrm{Ny}}=4,8$ 

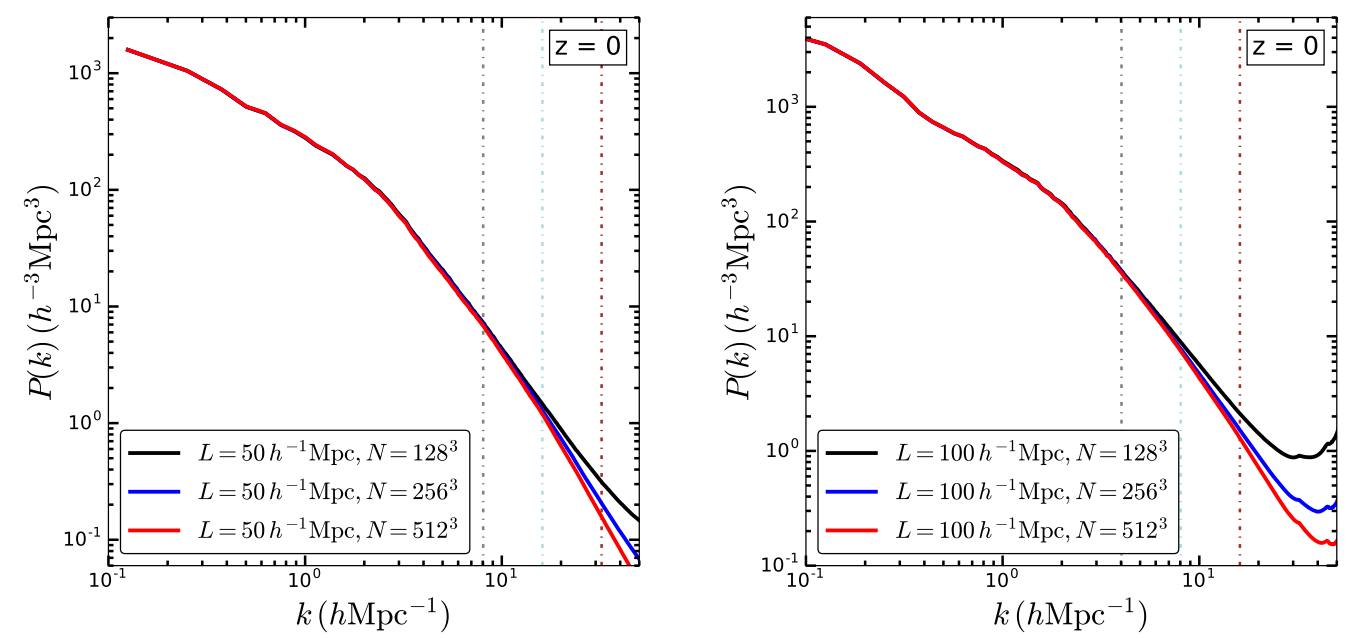

Figure 3. The matter power spectrum at $z=0$ of different simulation resolutions with $V=$ $50^{3} h^{-3} \mathrm{Mpc}^{3}$ (left panel) and $V=100^{3} h^{-3} \mathrm{Mpc}^{3}$ (right panel). One can see that in almost the entire range of scales the matter power spectra at different resolutions converge, all the way up to the Nyquist limit.

and $16 h \mathrm{Mpc}^{-1}$. On large and intermediate scales, the matter power spectra at different resolutions converge fairly well, starting to deviate beyond the Nyquist wavenumber $k_{\mathrm{Nyq}}$ of the given resolution. In particular, the excess of power above the Nyquist wavenumber is a manifestation of particle shot noise. In order to better highlight this effect, we show in the two panels of Fig 4 the ratios between each of the spectra and a (third) reference spectrum $P_{512}(k)$ for the $N=512^{3}$ case, evaluated at $z=0$. It can be seen that the simulations agree to within $5 \%$ or better below the Nyquist wavenumber. In particular, at $k=k_{\mathrm{Ny}} / 2$ the error at $z=0$ is $5.7 \%$ for the $128^{3}$ particles run and $3.4 \%$ for the $256^{3}$ run, for a box size of $50 h^{-1} \mathrm{Mpc}$. The corresponding numbers for the $100 h^{-1} \mathrm{Mpc}$ boxsize are $2.5 \%$ and $2.6 \%$.

From the results presented in this section, it is clear that the parameter set $V=$ $50^{3} h^{-3} \mathrm{Mpc}^{3}$ and $N=512^{3}$ provides an adequate benchmark choice for our simulations. In particular, we find that our simulations have $\sim 10 \%$ accuracy or better in the wavenumber range $(1-20) h \mathrm{Mpc}^{-1}$.

\section{Simulation Results}

By comparing the results of our simulations, we can infer the effect of DWDM on structure formation. In the following, we derive our results through detailed analyses of the density field, the matter power spectrum and the halo mass function inferred from our N-body simulations. 

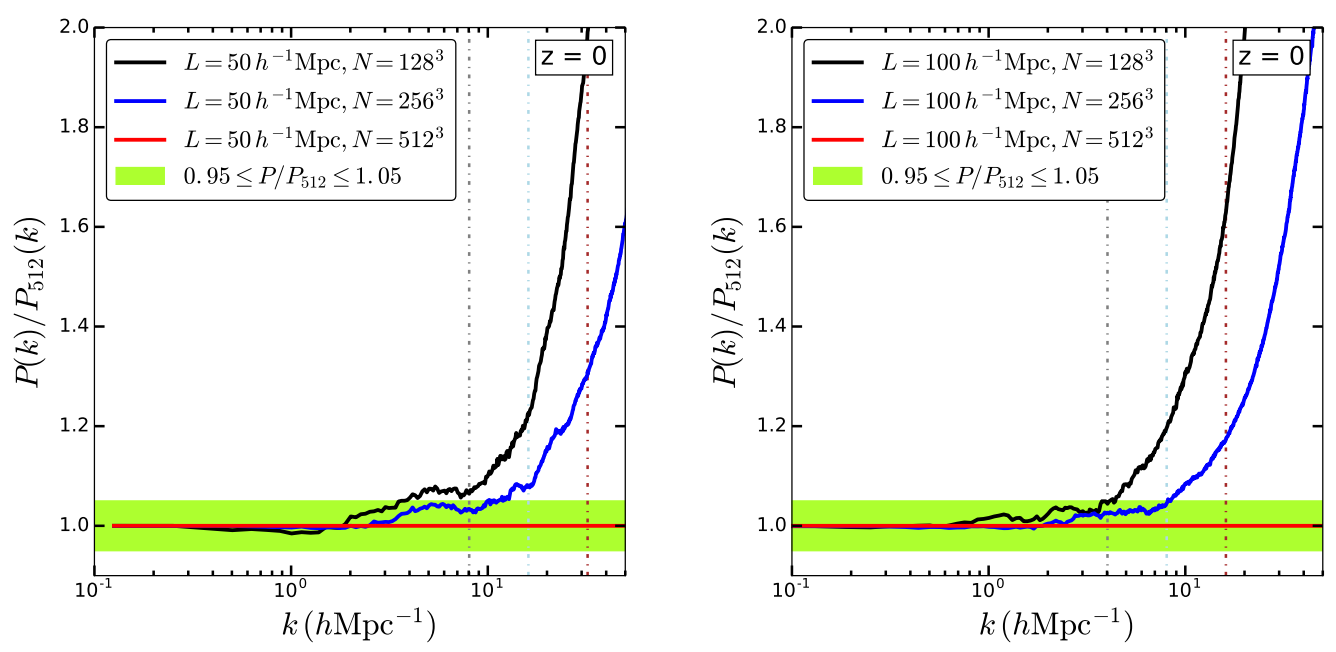

Figure 4. Effect of changing the simulation resolution at fixed box size $L$. The solid curves show the ratio between the matter power spectra at $z=0$ of Fig. 3, obtained with the settings for the particle number indicated in the legend, and the spectrum for $N=512^{3}$ choosen as reference. The left (right) panel is for $L=50(100) h^{-1} \mathrm{Mpc}$.

\subsection{Density Field}

In Fig. 5, we compare the density field extracted from different simulations. The first, second and third columns correspond to three different scenarios: CDM, WDM with mass $m_{J}=$ $1.5 \mathrm{keV}$ and WDM with mass $m_{J}=0.158 \mathrm{keV}$. The density field is calculated from the particle distribution by using the triangular shaped cloud scheme and further smoothed by a Gaussian filter. The first and second rows in Fig. 5 correspond to the stable and the decaying case. The density contrast $\delta$ is defined as

$$
\delta=\frac{\rho}{\bar{\rho}}-1
$$

where $\rho$ is the local density and $\bar{\rho}$ is the average density. The color scale we use in Fig. 5 is the logarithm of $\delta+1$, which represents the ratio of local density $\rho$ to the average density $\bar{\rho}$. With the density field and the color scale, one can see how different cosmic structures form in different cosmologies. By comparing the stable $\Lambda$ CDM and the stable WDM simulations, one can clearly see the suppression of structure in the SWDM case, due to the associated free-streaming effect. However, the effect of decay is not obvious through a simple visual comparison of the corresponding stable and decaying density fields.

The well-known suppression of small-scale structure characteristic of WDM is evident when comparing the different columns in Fig. 5 . We can see that a large portion of small-scale 

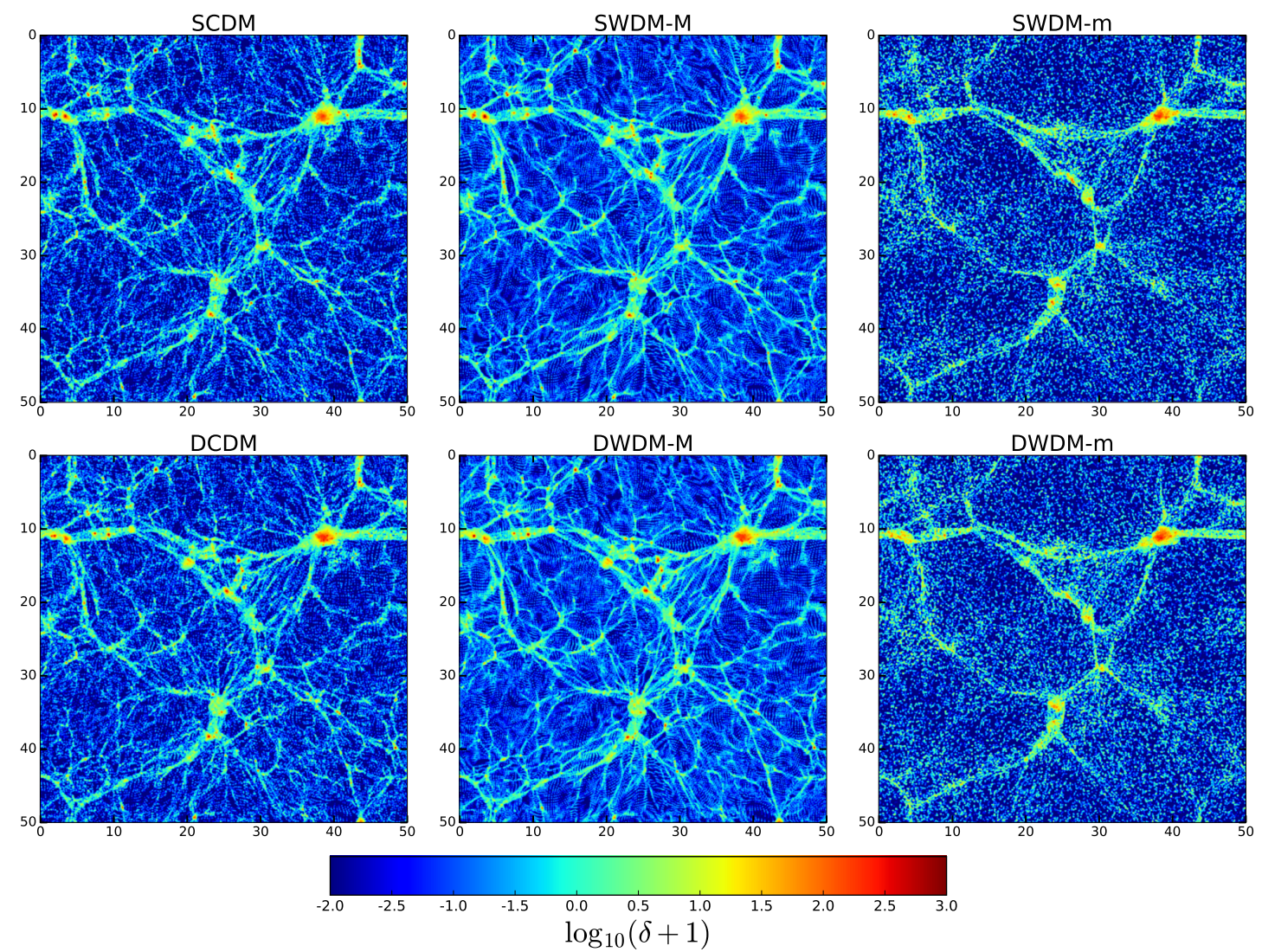

Figure 5. Comparison of the density fields at $z=0$. The first and second rows correspond to stable and decaying cases (with lifetime fixed at the CMB limit, 50 Gyr [21]), respectively. The first, second and third columns correspond to three different paradigms: $\Lambda \mathrm{CDM}$, and WDM with masses $m_{J}=1.5 \mathrm{keV}$ and $m_{J}=0.158 \mathrm{keV}$, from left to right. The horizontal and the vertical axis are given in units of $h^{-1} \mathrm{Mpc}$ and represent the size of the simulation box. One clearly sees the free-streaming effect of WDM, indicated by the suppression of structure in the density field of the WDM simulations.

structure is smoothed out in the WDM simulation with WDM mass $m_{J}=0.158 \mathrm{keV}$, due to the large free-streaming length. In fact, the free streaming wavenumber is only one order of magnitude larger than the fundamental mode of the box. On the other hand, the density fields of the $\Lambda$ CDM simulations and those of the WDM simulations with WDM mass $m_{J}=1.5 \mathrm{keV}$ look quite similar, because on the scales probed by the simulations free streaming is rather weak for such cases. However, a lack of small-scale power in the WDM simulation can still be observed.

Note that the small peaks in the density field of WDM simulations are due to spurious halos from finite resolution effects and numerical fragmentation, as discussed in Sec. 2.3. We will also discuss such effects on the halo mass function in Sec. 3.3.

It is difficult to appreciate the effect of decay by performing a quick visual comparison of 

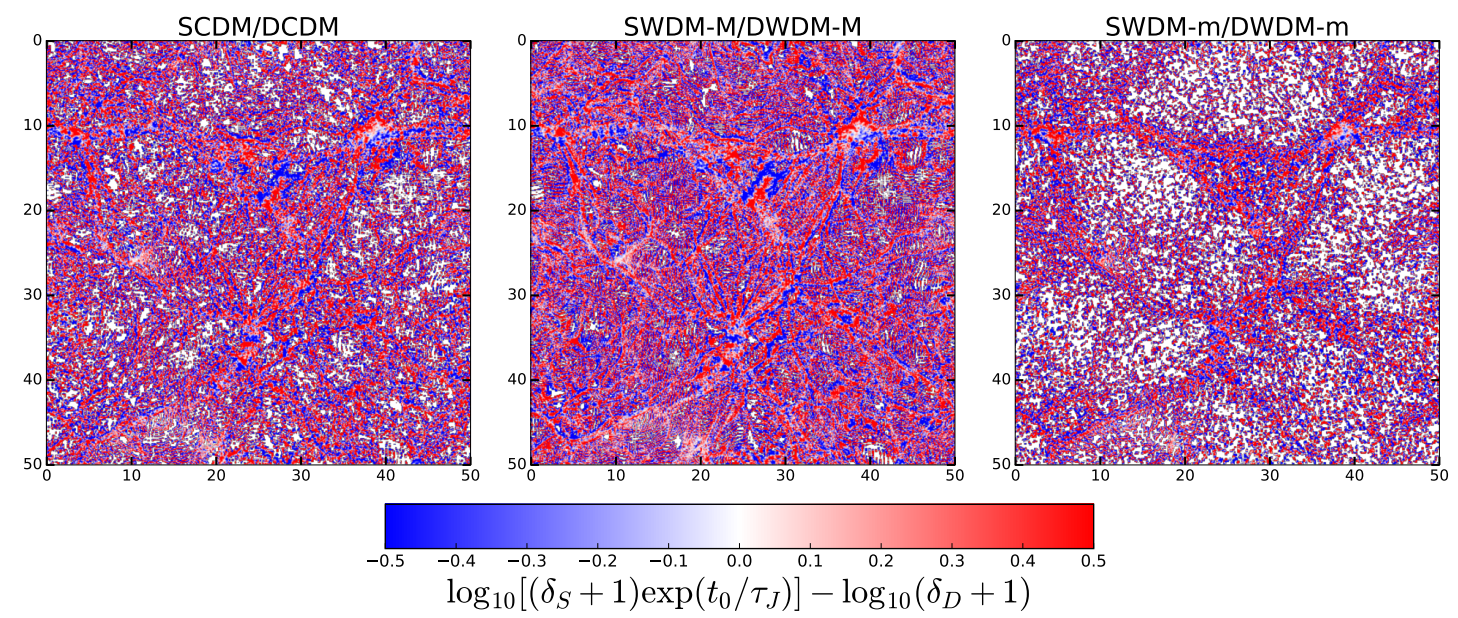

Figure 6. Comparison of the relative density fields at $z=0$. The left figure represents the relative density field of SCDM and DCDM, the middle figure of is the relative density field of SWDM-M and DWDM-M, and the right figure is the relative density field of SWDM-m and DWDM-m, respectively. The horizontal and vertical axis are given in the same units as in Fig. 5. One sees that in most of the regions, the density is larger in the stable case. However, there are small changes due to subtle features of the decay scenario, see text for explanation.

the density fields in Fig. 5. Thus, in order to better isolate the effect of decay, we refer to the relative density field $\rho_{S} / \rho_{D}$ of the stable (S) over the decaying (D) case, shown in Fig. 6. In that figure, the color scale refers to $\log _{10} \rho_{S} / \rho_{D}=\log _{10}\left[\left(\delta_{S}+1\right) \exp \left(t_{0} / \tau_{J}\right)\right]-\log _{10}\left(\delta_{D}+1\right)$. One can see that the decay effect reduces the density in most regions of the density field, especially near the center of halos and the interior of filaments. This follows from the change in the gravitational potential due to the decay, which makes the potential wells more shallow. This is reflected in the fact that most regions are (relatively) overdense in the stable case, as indicated by the reddish regions in Fig. 6. Note also that changes in the gravitational potential also affect the dynamics of the simulation, causing diffusion of the simulation particles. This makes the final density distribution more diffuse with respect to the stable case. Therefore, we can see that regions near the periphery of the halos and filaments are denser in the decaying DM case, and appear as the blu-ish regions in Fig. 6.

\subsection{Matter Power Spectrum}

The matter power spectrum of the simulations is calculated using the ComputePk code [38] with the triangular shaped cloud scheme. Note that, since in the simulation we neglect the decayproduced neutrinos, we only consider the overdensity of the DM and baryons in calculating the matter power spectrum. In Fig. 7 , we show the matter power spectrum at $z=\{0,1,2,3\}$ for each of the simulations that we have performed, focusing on the differences between stable and 

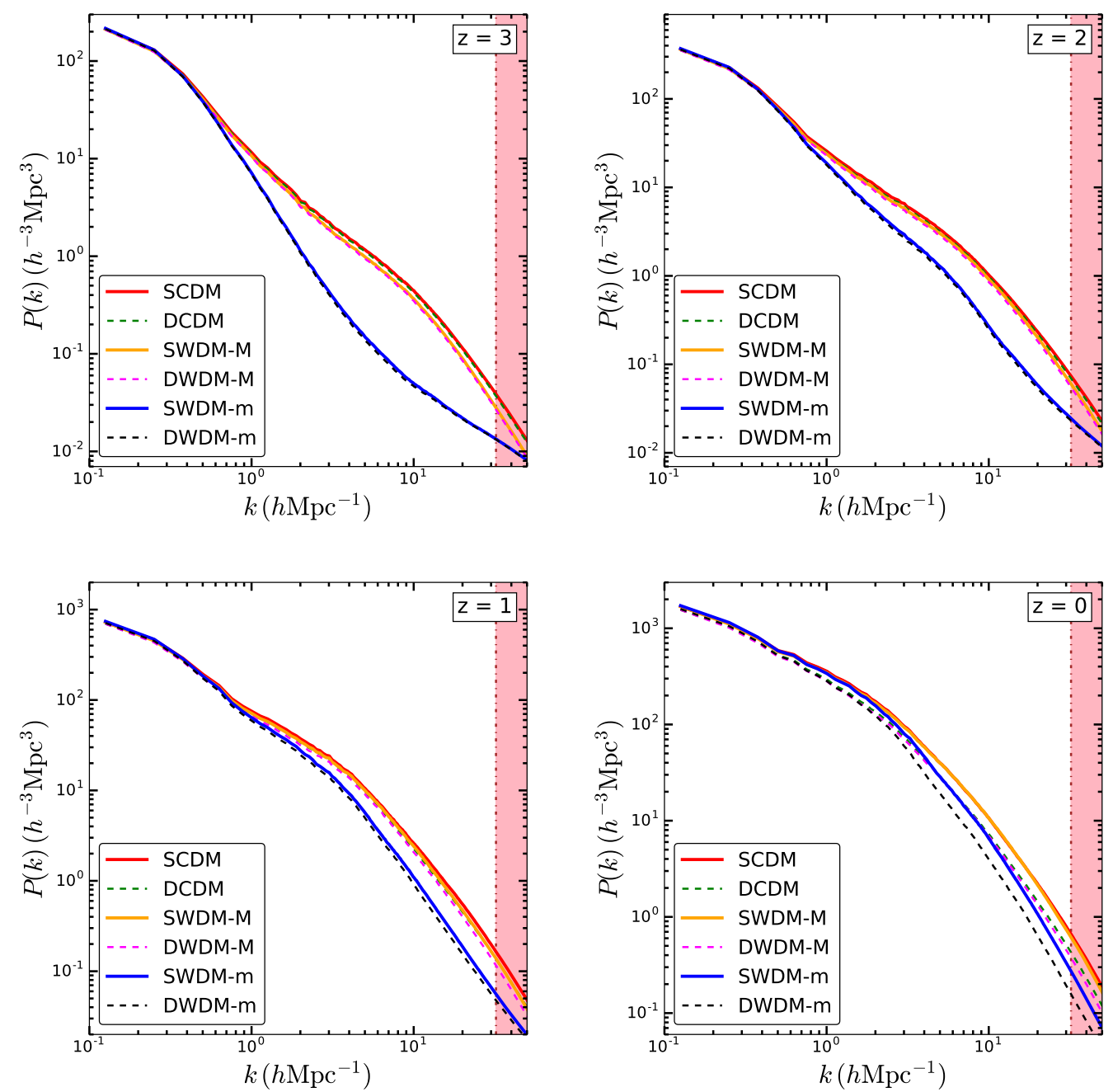

Figure 7. Matter power spectra derived from our simulations, for the standard $\Lambda$ CDM $\equiv$ SCDM, DCDM, SWDM-M, DWDM-M, SWDM-m and DWDM-m cases, at redshifts $z=0,1,2,3$. The solid lines represent the stable case, while the dashed ones correspond to the decaying case. The different colors are associated to different DM mass, and the pink band represents length scales smaller than the Nyquist limit. One can clearly see the evolution of the matter power spectrum, as well as late-time decay effects. Further details are given in the text.

decaying DM cases. The solid lines represent the matter power spectra from the simulations with stable DM, while the dashed lines correspond to the matter power spectra obtained in the simulations with decaying DM. The dashed vertical line corresponds to the Nyquist wavenumber $k_{\mathrm{Nyq}}$ defined in Eq. (2.6), i.e. the scale of the average interparticle distance the resolution limit of our simulations. For our simulation parameters, $k_{\mathrm{Nyq}} \simeq 32 \mathrm{~h} \mathrm{Mpc}^{-1}$.

From Fig. 7, one can easily see that the effect of decay becomes manifest at lower 
redshifts. This is due to the late decay time of the DM candidate ${ }^{4}$. To quantify the overall effect of decay, we focus on the matter power spectrum at $z=0$. As a reference, the scale of non-linearity at $z=0$ is roughly $0.15 h \mathrm{Mpc}^{-1}$. By comparing SWDM-m and SWDM-M with the standard $\Lambda \mathrm{CDM} \equiv \mathrm{SCDM}$ paradigm, one can see that the matter power spectra on large scales (small $k$ ) are identical, but differ on small scales, the SWDM spectra being suppressed due to the free-streaming effect of WDM. This effect is very evident for the SWDM-m case, that has the larger free-streaming length. The difference between SWDM-M and SCDM is instead small, and visibile only at the largest k's, because the free-streaming length for WDM with $m_{J}=1.5 \mathrm{keV}$ is still quite small, and thus free-streaming does not cause too much suppression on the scales probed in our simulations. By comparing DWDM-m and DWDMM with SCDM, one can see that the small scale suppression due to the free-streaming effect of WDM still exists. Moreover, there is further suppression on all scales caused by the effect of decay. The presence of the decay, inherent in the BV model [20], reduces the matter energy density in the universe, hence the growth factor is reduced, which delays the formation of structure. The decay-induced suppression does not show a strong dependence on scale. This should contrasted with the free-streaming effect of WDM, that has a strong dependence on the scale, due to the scale of the cut-off in the initial transfer function, related to the mass and temperature of the WDM. A lighter thermal WDM will cause a cut-off in the matter

\footnotetext{
${ }^{4}$ In our DWDM picture the late majoron decays simply reflect the tiny neutrino mass [16].
}
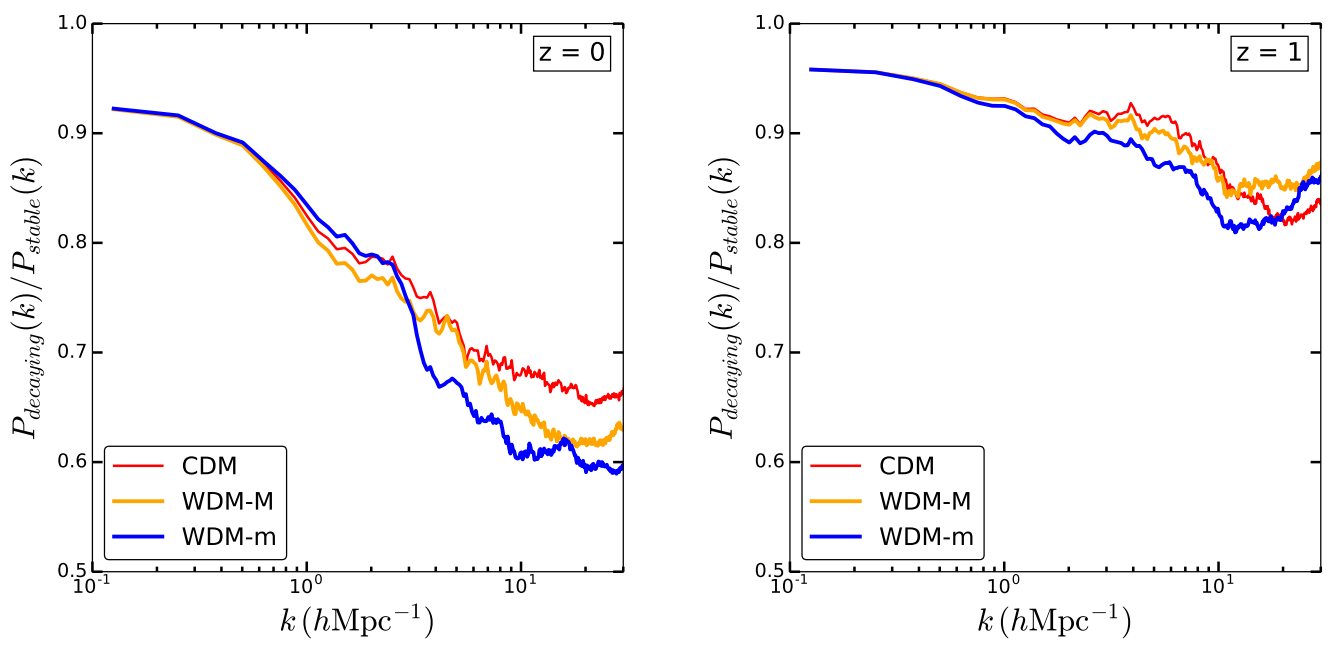

Figure 8. Left panel: Ratio between the matter power spectra of decaying and stable dark matter, for CDM (red), WDM-M (orange), WDM-m (blue) at $z=0$. Right panel: Same as the left panel, at $z=1$. It can be noticed how the effect of the decay is manifest on all scales, but is more evident on small scales, and also more evident for WDM with respect to CDM. 
power spectrum on a larger scale, as originally envisaged in the BV model.

The suppression due to the decay seems however to gradually decrease towards large scale. To better assess this behaviour, we compute the ratio between the decaying and stable power spectra, $P_{\text {decaying }}(k) / P_{\text {stable }}(k)$, for each of the three pairs of simulations. These are shown, for $z=0$ and $z=1$, in Fig. 8, that can be compared with Fig. 2 in Ref. [31], that shows the same quantity for the CDM case. First of all, we note that our results for CDM are very consistent with those of Ref. [31], also considered the slighthly different values for the lifetimes between their work and the present one. Let us briefly discuss the features of the curves in Fig. 8. We find first of all confirmation that the decay suppresses the spectrum on all scales under consideration, since the curves always lie below unity. On the largest scales shown in the plot, the three ratios converge to a constant common value. That the large-scale behaviour of the curves is common could be easily expected by noting that, above the freestreaming length, WDM and CDM behave the same way. On the other hand, the suppression due to the decay is more evident on small scales, in all the cases under consideration. As noted by Enqvist et al. [31], this is due to the fact that mode-mode couplings make that differences that are small in the linear regime get enhanced by the nonlinear evolution. In fact, we can see that this effect is less pronounced in the right panel of Fig. 8, corresponding to $z=1$, when more scales were still in the linear regime with respect to $z=0$ (shown in the left panel).

Another interesting feature that can be noticed in Fig. 8 is that the nonlinear enhancement of the effect of the decay on small scales is stronger for lighter WDM. There is a distinct drop in the curve, especially evident in the $m_{J}=0.158 \mathrm{keV}$ case, at scales right above the free-streaming length. In other words, it seems that the combination of the cutoff in the linear power spectrum due to the WDM thermal velocity and of the nonlinear evolution, enhances the effect of the DM decay.

To summarize, by comparing the decaying and the stable cases, one can see that the effect of decay is to suppress power on all scales, with the suppression being more severe on the small, nonlinear scales. In contrast, the effect of WDM per se is to suppress the matter power spectrum on small scales, depending on the mass of the WDM candidate. Also, the small-scale suppression due to the decay is more evident for WDM, as one can see by comparing the matter power spectrum of $\Lambda \mathrm{CDM}$ simulations with those corresponding to WDM simulations. 

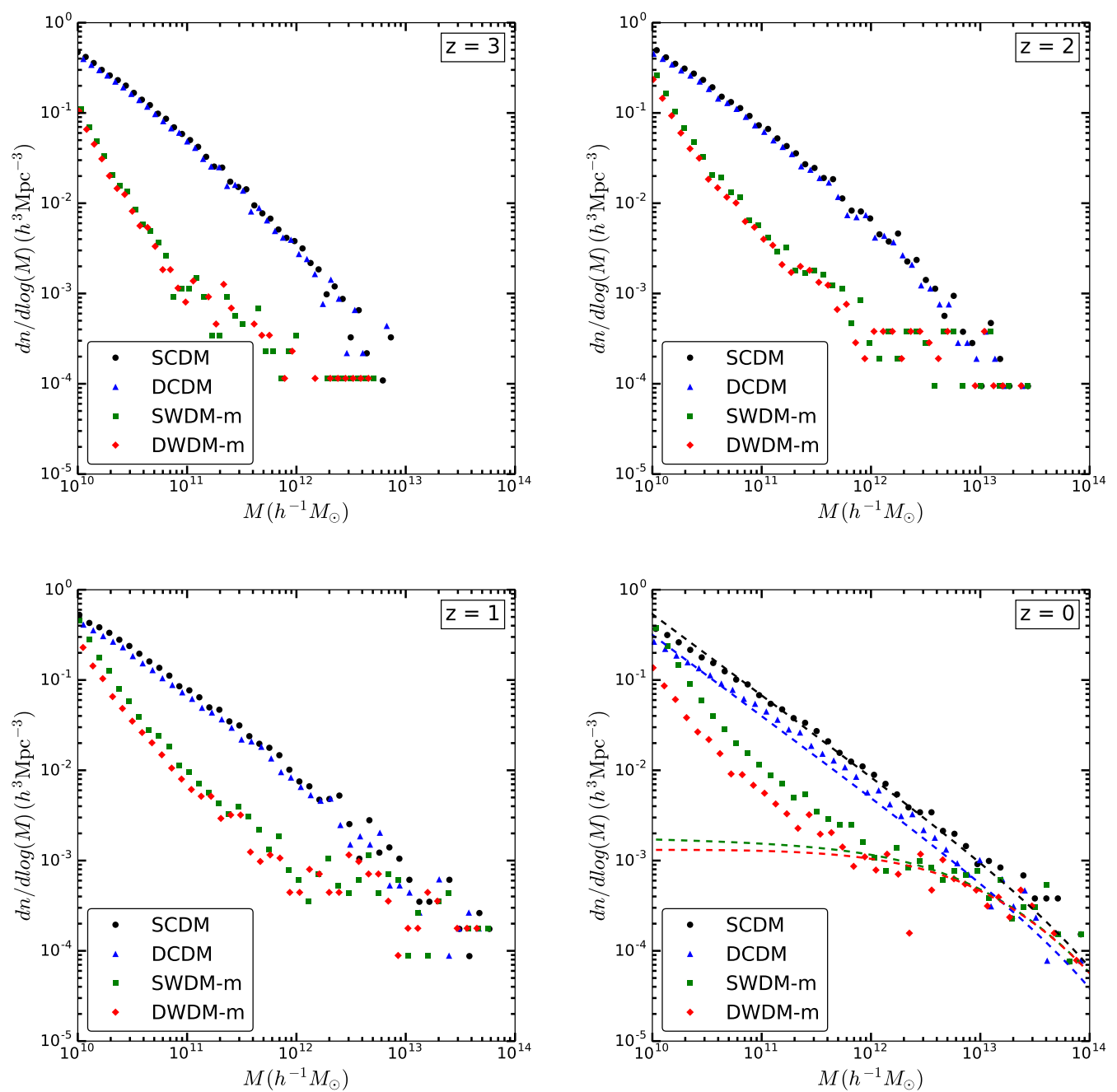

Figure 9. Evolution of the halo mass function for the standard $\Lambda$ CDM $\equiv$ SCDM paradigm (black circle) compared with the simulations corresponding to DCDM (blue triangle), SWDM-m (green square), and DWDM-m (red diamond). The dashed lines of corresponding colors in the $z=0$ panel represent our derived halo mass function fits based on the given cosmology and the data points obtained from our simulations. The data points of our simulations that do not fit well to the theoretical WDM halo mass function are mainly due to spurious halos. By comparing the stable and decaying cases, we can see that the effect of decay is to reduce the number density of halos for all mass scales. However, the effect of the warm DM nature is seen by setting a cut-off mass, which is the mass scale that the halo mass functions of WDM simulations start to deviate from those of CDM simulations.

\subsection{Halo Mass Function}

The halo mass function is defined as the number density of DM halos per unit logarithmic mass interval. In order to estimate the halo mass function, we need to identify halos, i.e., bound objects, within the large set of particles in our simulations. For this purpose, we make use of 
the parallel halo finder package AHF [39] in order to calculate the halo mass function based on our snapshots of simulations. In AHF, the adaptive mesh refinement algorithm is adopted to identify clumps in the density field. Therefore, it can build up the hierarchical structure for the halos and sub-halos obtained in the snapshots. After iteratively removing the particles unbounded by the gravitational potential of the halo and refining the halo edge, the properties of the halos are finally determined by the particles within its virial radius $R_{v i r}$. Here $R_{v i r}$ is defined as the point where the density profile of the particles drops below $\Delta_{v i r} \rho_{c}$ in which $\Delta_{v i r}$ is a constant depending on the cosmology and the $\rho_{c}$ is the critical density of the universe. In Fig. 9, the black circle, blue triangle, green square, and red diamond represent halo mass functions obtained from the $\Lambda \mathrm{CDM} \equiv \mathrm{SCDM}$ paradigm and the simulations corresponding to DCDM, SWDM-m and DWDM-m, respectively. In order to avoid cluttering in the figures, we do not illustrate the halo mass functions corresponding to DWDM-M and SWDM-M in the figure. The dashed lines of corresponding colors in the $z=0$ panel are the halo mass function fits calculated based on the given cosmology and the halo mass functions obtained from our simulations.

Note that as a result of the strong cut-off in the WDM transfer function, resulting in a suppression of small-scale power, discreteness effects close to the resolution limit will be more important than for CDM simulations. Indeed, as a consequence of finite resolution effects in the simulations, spurious clumps are produced by numerical fragmentation [37, 40, 41]. Note that the halos produced in early simulations [33] were considered to be the result of the "top-down" structure formation scenario of WDM [42]. However, further studies have demonstrated that this phenomenon depends on the average interparticle distance, i.e. on the resolution of the simulation, hence could be regarded as a numerical artifact [37]. For small halo masses, these spurious clumps will outnumber the genuine halos.

In order to identify the spurious clumps, we first calculate the halo mass function of the corresponding cosmology using the code hmf, which is the back end of HMFcalc [43], with the fitting model in Ref. [44]. Then we introduce the fitting method in Ref. [45], which provides a precise fit for the halo mass function of WDM cosmology. The overall halo mass function fit for the WDM scenarios used in this work can be written as

$$
n(M)=\left(1+M_{h m} / M\right)^{-\gamma} \times n_{\text {Tinker }}(M),
$$

where the $M_{h m}$ is defined as the mass scale at which the amplitude of the WDM transfer function is reduced to $1 / 2, \gamma$ is a free parameter for fitting the correct shape of the halo mass function and $n_{\text {Tinker }}(M)$ is the halo mass function fit in Ref. [44]. $M_{h m}$ is expected to mainly affect the properties of WDM haloes [45]. $M_{h m}$ is related to its corresponding length scale 
$\lambda_{h m}$ by

$$
M_{h m}=\frac{4 \pi}{3} \bar{\rho}\left(\frac{\lambda_{h m}}{2}\right)^{3},
$$

where $\bar{\rho}$ is the average density of the universe. Here we follow Ref. [45] to calculate $M_{h m}$ based on our simulation results and perform the fitting. For the best-fit values, we find that $\gamma \approx 0.309$ in SWDM-m and $\gamma \approx 0.345$ in DWDM-m, in which the larger $\gamma$ for DWDM-m indicates the existence of further suppression coming from the decay. For the halo mass function fit for DCDM, we simply introduce a factor $A$ to account for the effect of decay, which is written as $n(M)=A \times n_{\text {Tinker }}(M)$.

By comparing the halo mass function fits with the halo mass functions from the simulations, we can infer that for SWDM-m and DWDM-m the halo mass functions from the simulations (green square and red diamond) deviating from the corresponding halo mass function fits are mainly composed of spurious halos. The genuine halo mass functions for WDM simulations should exhibit the same trend as the corresponding halo mass function fits for small masses. The effect of decay and the free-streaming effect of WDM can also be separated due to their distinct impact on the halo mass functions. From the difference between the stable case and decaying case, we can infer that the effect of decay in the halo mass function is to reduce the number density of halos in every mass scale. In other words, the effect of decay produces an overall downward shift on the halo mass function. On the other hand, the free-streaming effect of WDM is to set a cut-off halo mass which is roughly the mass scale that the WDM halo mass function deviates from CDM halo mass function. We also note that, at large halo masses, it is difficult to assess the differences between the halo mass functions of different cosmologies, due to the variance caused by the scarcity of halos with large masses.

\subsection{Effects of baryonic physics}

To close our discussion we now comment on the effects of baryonic physics, so far neglected. Despite the fact that baryons are themselves biased tracers of the DM gravitational potential, their role in structure formation is distinct from that of DM as a result of their ability to cool down through radiative processes. This makes baryons able to form compact astrophysical objects, such as stars, resulting in a different distribution compared to that of DM. Thanks to the development of N-body simulation techniques, high-resolution and large-scale hydrodynamics simulations have now become feasible. Many studies have shown that baryonic processes can generate non-trivial effects on astrophysical observables such as the halo density profile, the matter power spectrum and the halo mass function. However, the precise details of baryonic processes remain poorly understood [35, 46-51]. Baryons affect the halo mass and density profile in several ways. When falling into the potential wells created by 
the DM, baryons are gravitationally heated and exchange energy with DM during relaxation. Hence they remain more diffuse and can create core-like density profiles at the center of halos. Later, as they dissipate energy through radiative processes, baryons sink into the center of halos and finally convert into stars. This steepens the density profile near the center. On the other hand, the presence of supernovae (SN) and active galactic nuclei (AGN), reduces the effect of radiative cooling and adiabatic contraction, since baryons are ejected out from the center of the halos. Baryonic physics also affects the matter power spectrum, see for example Ref. [35] for a comprehensive review. At intermediate scales $\left(k \simeq 0.8-5 h \mathrm{Mpc}^{-1}\right)$ the power spectrum is suppressed because the pressure of baryons smoothens the density field. In contrast, the spectrum rises at small scales because of radiative cooling that allows baryons to cluster at those scales. Concerning the halo mass function, the number density of low-mass halos increases as a result of cooling and star formation. However, AGN feedback can reduce the effect of cooling and hence the abundance of low-mass halos.

In summary, we have shown that in a DDM cosmology, there is an overall suppression of the density fluctuations, due to the decay of the DM particle. This is accompanied, in the case of WDM, by the well-known smoothing of the density field and suppression of the small-scale power in the matter power spectrum and halo mass function. Taking into account the full set of baryonic processes, including gravitational heating, radiative cooling, adiabative contraction, stellar and AGN feedback, we expect that the impact of baryonic physics will be generally weakened by the decaying and warm nature of DM. In fact, since the gravitational potential is shallower in the DWDM scenario, due to both the decay and the free-streaming of WDM, the ability of halos to accrete baryons into their center will be somehow limited. Therefore, we expect the star-formation rate to decrease as a result, leading in turn to a lower efficiency of the stellar and AGN feedback. In this sense, we would argue that the baryon component remains in a relatively smooth density distribution containing fewer structures, while still being a biased tracer of the DM. This is only a qualitative assessment of the effects of baryonic physics in a DWDM structure formation scenario. Full-fledged hydrodynamic simulations would be necessary to assess the interplay between baryons and DWDM.

\section{Conclusions}

In this paper we have examined the cosmology of warm dark matter, both for the stable as well as decaying cases, paying special attention to how it affects structure formation. We have performed DM-only N-body simulations of the nonlinear evolution and compared the matter power spectrum associated to warm dark matter masses of $1.5 \mathrm{keV}$ and $0.158 \mathrm{keV}$, with that expected for the stable cold dark matter $\Lambda$ CDM paradigm, taken as our reference model. We 
have scrutinized the effects associated to the warm nature of dark matter, as well as the fact that it decays. We find that the nonlinear evolution somehow couples the two effects, in such a way that the effect of the decay becomes more pronounced below the free-streaming scale of WDM. All of our considerations are general, though we have been strongly motivated by the fact that the DWDM scenario can naturally appear in particle physics. A nice example is provided by the $\mathrm{keV}$ majoron DM scenario suggested in the original BV-proposal [20]. The majoron itself emerges as a Nambu-Goldstone boson within a broad class of particle theories where neutrino mass generation takes place through the spontaneous breaking of a continuous ungauged lepton number symmetry. The majoron picks up a mass from gravitational effects, expected to explicitly break global symmetries. Hence it must necessarily decay to neutrinos, with an amplitude proportional to their tiny mass, which typically gives it cosmologically long lifetimes [16]. As a reference value for decaying dark matter lifetime we have taken the conservative limit following from CMB observations obtained in [22]. We have modified the standard N-body simulation code so as to include the effect of decay besides the free-streaming effect. Through these simulations we have shown that the DWDM picture suggested in the BV proposal leads to predictions on small scales that differ substantially from those of the standard $\Lambda \mathrm{CDM}$ paradigm. A dedicated analysis, using better resolution simulations and including baryons, is required to assess whether this could address the potential drawbacks of the $\Lambda \mathrm{CDM}$ scenario. We have also qualitatively discussed the possible impact on the DWDM scenario when baryonic physics is taken into account. Our results illustrate that the observations of large-scale structures in the Universe can in principle be used in order to constrain the particle physics model underlying the origin of dark matter. In our case, our results may be extended in order to constrain the lifetime and mass of the $\mathrm{keV}$ dark matter majoron.

\section{Acknowlegdments}

This research was supported by the Spanish grants FPA2017-85216-P (AEI/FEDER, UE), SEV-2014-0398 and PROMETEOII/2018/165 (Generalitat Valenciana), by the Italian INFN through the InDark and Gruppo IV fundings, by ASI through the Grant 2016-24-H.0 (COSMOS) and the ASI/INAF Agreement I/072/09/0 for the Planck LFI Activity of Phase E2, and by the Taiwan MoST grants MOST-105-2112-M-007-028-MY3 and MOST-107-2112-M007-029-MY3. 


\section{A Results for $5.3 \mathrm{keV}$ decaying dark matter}

Although the uncertainty in the evolution of the IGM temperature might cast doubt on the interpretation of the Lyman-alpha forest $[52,53]$ measurements, we note that recent Lymanalpha forest observations may set a strong lower limit on the WDM mass. Therefore, for completeness, we also perform simulations using the 95\% CL lower limit on the mass of the WDM particle allowed by Lyman-alpha forest [26] data, i.e. $m_{J} \geq 5.3 \mathrm{keV}$. We keep the lifetime $\tau_{J}=50 \mathrm{Gyr}$ as in the other simulations with decay. In this appendix, we present the results with such a mass for both stable and decaying dark matter.

In Fig. 10, we compare the matter power spectrum of SWDM and DWDM with $m_{J}=$ $5.3 \mathrm{keV}$ to that of SCDM. We show the individual matter power spectra at $z=0$ in the left panel, and the ratios to the SCDM matter power spectrum in the right panel. Note that the difference between SWDM with $m_{J}=5.3 \mathrm{keV}$ and SCDM is smaller than $1 \%$ on all scales. This is associated to the relatively small free-streaming length of such a "large" mass WDM particle. Furthermore, a visual comparison with the red solid curve in the left panel of Fig. 8, shows that the power suppression due to the decay is in practice the same for WDM with $m_{J}=5.3 \mathrm{keV}$ and DCDM. This is again a consequence of the small free-streaming length of the WDM.

Similar considerations apply to the halo mass functions for SCDM, SWDM and DWDM,
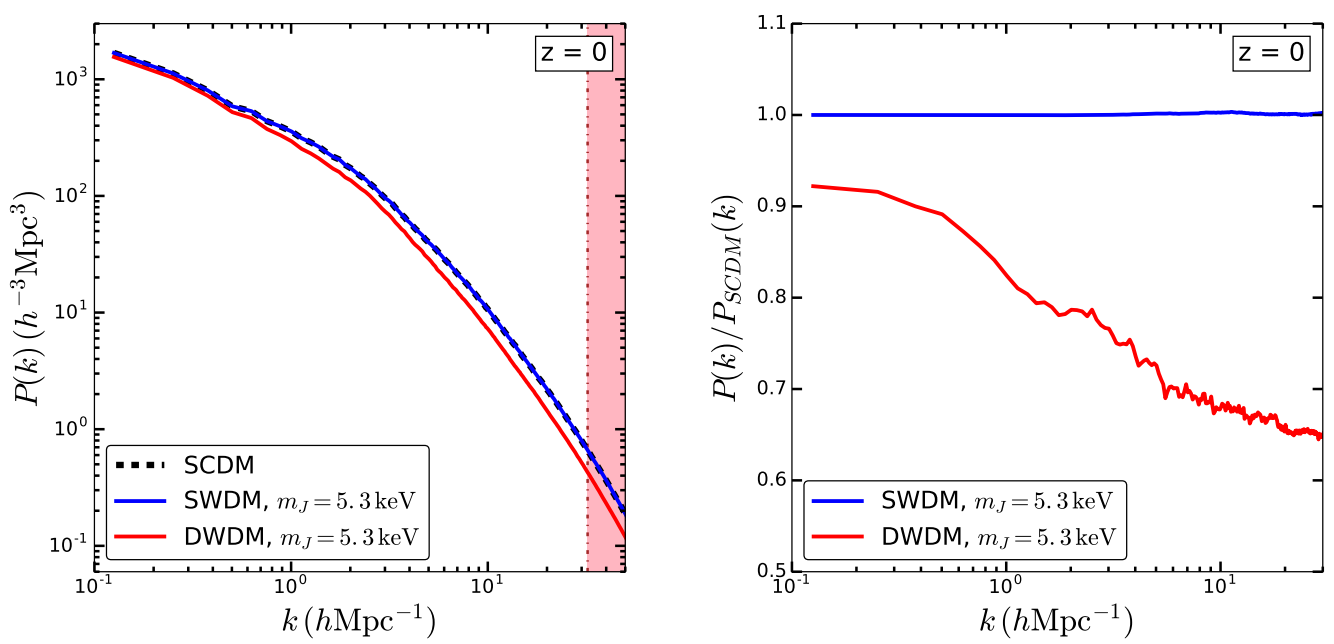

Figure 10. Left panel: The matter power spectrum at $z=0$ for SCDM (black dashed), SWDM (blue solid) and DWDM (red solid) with $m_{J}=5.3 \mathrm{keV}$. Right panel: Ratio between SWDM (blue solid) and DWDM (red solid) with $m_{J}=5.3 \mathrm{keV}$ and SCDM. The ratio between SWDM and SCDM is very close to 1 on all scales, due to the small free-streaming length of such a heavy WDM particle. 
shown in Fig. 11. The number densities of halos are almost identical for SCDM and SWDM with $m_{J}=5.3 \mathrm{keV}$, except for some deviations in the high-mass end due to cosmic variance. Also note that the large number of spurious halos that were seen in the light WDM simulations discussed in Sec. 3.3 disappear for WDM with $m_{J}=5.3 \mathrm{keV}$. Moreover, the decay suppresses the halo mass function of DWDM on all scales.

From the analysis of the matter power spectrum and the halo mass function, we conclude that the WDM mass allowed by the Lyman-alpha forest is, at the scales probed by our analysis, undistinguishable from CDM. This holds for both the stable and decaying case.

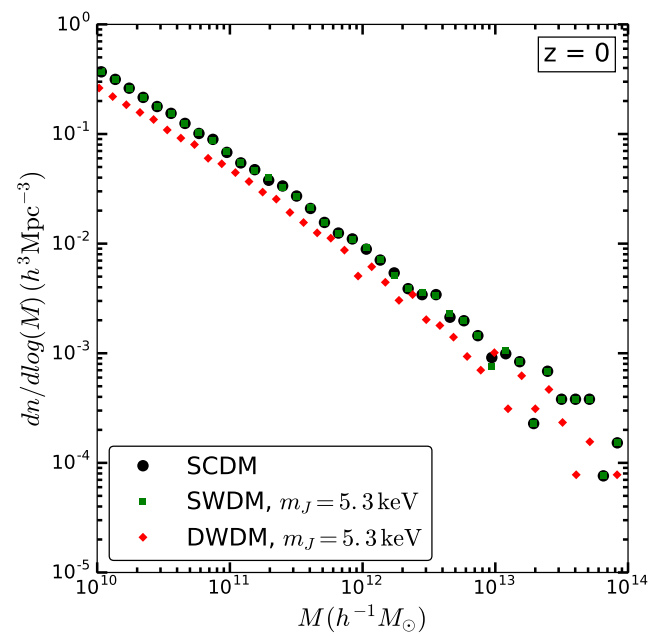

Figure 11. The halo mass function at $z=0$ for SCDM (black circle), SWDM (green square) and DWDM (red diamond) with $m_{J}=5.3 \mathrm{keV}$. Like the matter power spectrum, the halo mass function is similar for SCDM and SWDM with $m_{J}=5.3 \mathrm{keV}$, despite for some deviations in the high-mass end related to cosmic variance. The halo mass function of DWDM with $m_{J}=5.3 \mathrm{keV}$ shows suppression of the halo number density compared to that of SCDM and SWDM at all mass scales, as discussed in Sec. 3.3. 


\section{References}

[1] G. Bertone, D. Hooper, and J. Silk, "Particle dark matter: evidence, candidates and constraints," Physics Reports 405 no. 5-6, 279-390.

http://www.sciencedirect.com/science/article/pii/S0370157304003515. .

[2] T. Kajita, "Nobel Lecture: Discovery of atmospheric neutrino oscillations," Rev. Mod. Phys. 88 no. 3, (2016) 030501.

[3] A. B. McDonald, "Nobel Lecture: The Sudbury Neutrino Observatory: Observation of flavor change for solar neutrinos," Rev. Mod. Phys. 88 no. 3, (2016) 030502.

[4] J. W. F. Valle and J. C. Romao, Neutrinos in high energy and astroparticle physics. John Wiley \& Sons (2015), www.wiley.com/buy/9783527411979. ISBN 978-3-527-41197-9.

[5] M. Lattanzi, R. A. Lineros, and M. Taoso, "Connecting neutrino physics with dark matter," New J. Phys. 16 no. 12, (2014) 125012, arXiv:1406.0004 [hep-ph].

[6] E. Ma, "Verifiable radiative seesaw mechanism of neutrino mass and dark matter," Phys.Rev. D73 077301, arXiv:hep-ph/0601225 [hep-ph].

[7] M. Hirsch et al., "WIMP dark matter as radiative neutrino mass messenger," JHEP 1310 149, arXiv:1307.8134 [hep-ph].

[8] A. Merle et al., "Consistency of WIMP Dark Matter as radiative neutrino mass messenger," JHEP 07 (2016) 013, arXiv:1603.05685 [hep-ph].

[9] C. Bonilla, E. Ma, E. Peinado, and J. W. F. Valle, "Two-loop Dirac neutrino mass and WIMP dark matter," Phys. Lett. B762 (2016) 214-218, arXiv:1607.03931 [hep-ph].

[10] S. Centelles Chuliá et al., "Dirac Neutrinos and Dark Matter Stability from Lepton Quarticity," Phys. Lett. B767 209-213, arXiv:1606.04543 [hep-ph].

[11] M. Hirsch et al., "Discrete dark matter," Phys.Rev. D82 116003, arXiv:1007.0871 [hep-ph].

[12] M. Boucenna et al., "Predictive Discrete Dark Matter Model," Phys. Rev. D86 (2012) 073008, arXiv:1204.4733 [hep-ph].

[13] D. Restrepo et al., "Gravitino dark matter and neutrino masses with bilinear R-parity violation," Phys.Rev. D85 023523, arXiv:1109.0512 [hep-ph].

[14] K.-Y. Choi, D. E. Lopez-Fogliani, C. Munoz, and R. R. de Austri, "Gamma-ray detection from gravitino dark matter decay in the mu nu SSM," JCAP 1003 028, arXiv:0906.3681 [hep-ph].

[15] Y. Chikashige, R. N. Mohapatra, and R. D. Peccei, "Are there real Goldstone bosons associated with broken lepton number?," Phys. Lett. B98 26.

[16] J. Schechter and J. W. F. Valle, "Neutrino Decay and Spontaneous Violation of Lepton Number," Phys. Rev. D25 (1982) 774. 
[17] D. H. Weinberg, J. S. Bullock, F. Governato, R. Kuzio de Naray, and A. H. G. Peter, "Cold dark matter: controversies on small scales," Proc. Nat. Acad. Sci. 112 (2014) 12249-12255, arXiv:1306.0913 [astro-ph.C0]. [Proc. Nat. Acad. Sci.112,2249(2015)].

[18] J. S. Bullock and M. Boylan-Kolchin, "Small-Scale Challenges to the $\Lambda$ CDM Paradigm," Ann. Rev. Astron. Astrophys. 55 (2017) 343-387, arXiv:1707.04256 [astro-ph.CO].

[19] S. R. Coleman, "Why There Is Nothing Rather Than Something: A Theory of the Cosmological Constant," Nucl. Phys. B310 643.

[20] V. Berezinsky and J. W. F. Valle, "The KeV majoron as a dark matter particle," Phys.Lett. B318 (1993) 360-366, arXiv:hep-ph/9309214 [hep-ph].

[21] M. Lattanzi and J. W. F. Valle, "Decaying warm dark matter and neutrino masses," Phys. Rev. Lett. 99 121301, arXiv:0705.2406 [astro-ph].

[22] M. Lattanzi et al., "Updated CMB, X- and gamma-ray constraints on Majoron dark matter," Phys.Rev. D88 063528, arXiv:1303.4685 [astro-ph.HE].

[23] B. Audren, J. Lesgourgues, G. Mangano, P. D. Serpico, and T. Tram, "Strongest model-independent bound on the lifetime of Dark Matter," JCAP 1412 no. 12, (2014) 028, arXiv:1407.2418 [astro-ph.CO].

[24] V. K. Narayanan, D. N. Spergel, R. Dave, and C.-P. Ma, "Constraints on the mass of warm dark matter particles and the shape of the linear power spectrum from the Ly $\alpha$ forest," Astrophys. J. 543 (2000) L103-L106, arXiv:astro-ph/0005095 [astro-ph].

[25] J. Baur, N. Palanque-Delabrouille, C. Yèche, C. Magneville, and M. Viel, "Lyman-alpha Forests cool Warm Dark Matter," JCAP 1608 no. 08, (2016) 012, arXiv:1512.01981 [astro-ph.C0].

[26] V. Iršič et al., "New Constraints on the free-streaming of warm dark matter from intermediate and small scale Lyman- $\alpha$ forest data," Phys. Rev. D96 no. 2, (2017) 023522, arXiv:1702.01764 [astro-ph.C0].

[27] F. Bazzocchi et al., "X-ray photons from late-decaying majoron dark matter," JCAP 0808 (2008) 013, arXiv:0805.2372 [astro-ph]. * Brief entry *.

[28] A. Garzilli, A. Magalich, T. Theuns, C. S. Frenk, C. Weniger, O. Ruchayskiy, and A. Boyarsky, "The Lyman- $\alpha$ forest as a diagnostic of the nature of the dark matter," arXiv:1809.06585 [astro-ph.co].

[29] V. Springel, "The Cosmological simulation code GADGET-2," Mon. Not. Roy. Astron. Soc. 364 (2005) 1105-1134, arXiv:astro-ph/0505010 [astro-ph].

[30] Y. Suto, "Correlation function in decaying particle cosmology," Astrophys. J. 321 (1987) 36-48.

[31] K. Enqvist, S. Nadathur, T. Sekiguchi, and T. Takahashi, "Decaying dark matter and the tension in $\sigma_{8}$, , JCAP 1509 no. 09, (2015) 067, arXiv:1505.05511 [astro-ph.C0].

[32] D. J. Eisenstein and W. Hu, "Power spectra for cold dark matter and its variants," Astrophys. J. 511 (1997) 5, arXiv:astro-ph/9710252 [astro-ph]. 
[33] P. Bode, J. P. Ostriker, and N. Turok, "Halo formation in warm dark matter models," Astrophys. J. 556 (2001) 93-107, arXiv:astro-ph/0010389 [astro-ph].

[34] M. Crocce, S. Pueblas, and R. Scoccimarro, "Transients from Initial Conditions in Cosmological Simulations," Mon. Not. Roy. Astron. Soc. 373 (2006) 369-381, arXiv:astro-ph/0606505 [astro-ph].

[35] M. P. van Daalen, J. Schaye, C. M. Booth, and C. D. Vecchia, "The effects of galaxy formation on the matter power spectrum: A challenge for precision cosmology," Mon. Not. Roy. Astron. Soc. 415 (2011) 3649-3665, arXiv:1104.1174 [astro-ph.C0].

[36] S. Colombi, A. H. Jaffe, D. Novikov, and C. Pichon, "Accurate estimators of power spectra in N-body simulations," Mon. Not. Roy. Astron. Soc. 393 (2009) 511, arXiv:0811.0313 [astro-ph].

[37] J. Wang and S. D. M. White, "Discreteness effects in simulations of Hot/Warm dark matter," Mon. Not. Roy. Astron. Soc. 380 (2007) 93-103, arXiv:astro-ph/0702575 [ASTRO-PH].

[38] B. L'Huillier, "computePk: Power spectrum computation," Mar., 2014. in Astrophysics Source Code Library, record ascl:1403.015.

[39] S. R. Knollmann and A. Knebe, "Ahf: Amiga's Halo Finder," Astrophys. J. Suppl. 182 (2009) 608-624, arXiv:0904.3662 [astro-ph.C0].

[40] M. R. Lovell, C. S. Frenk, V. R. Eke, A. Jenkins, L. Gao, and T. Theuns, "The properties of warm dark matter haloes," Mon. Not. Roy. Astron. Soc. 439 (2014) 300-317, arXiv:1308.1399 [astro-ph.CO].

[41] M. Leo, C. M. Baugh, B. Li, and S. Pascoli, "The Effect of Thermal Velocities on Structure Formation in N-body Simulations of Warm Dark Matter," JCAP 1711 no. 11, (2017) 017, arXiv: 1706.07837 [astro-ph.CO].

[42] A. Knebe, J. E. G. Devriendt, B. K. Gibson, and J. Silk, "Top-down fragmentation of a warm dark matter filament," Mon. Not. Roy. Astron. Soc. 345 (2003) 1285, arXiv:astro-ph/0302443 [astro-ph].

[43] S. Murray, C. Power, and A. Robotham, "HMFcalc: An Online Tool for Calculating Dark Matter Halo Mass Functions," arXiv:1306.6721 [astro-ph.C0].

[44] J. L. Tinker, A. V. Kravtsov, A. Klypin, K. Abazajian, M. S. Warren, G. Yepes, S. Gottlober, and D. E. Holz, "Toward a halo mass function for precision cosmology: The Limits of universality," Astrophys. J. 688 (2008) 709-728, arXiv:0803.2706 [astro-ph].

[45] A. Schneider, R. E. Smith, A. V. Maccio, and B. Moore, "Nonlinear Evolution of Cosmological Structures in Warm Dark Matter Models," Mon. Not. Roy. Astron. Soc. 424 (2012) 684, arXiv:1112.0330 [astro-ph.CO].

[46] D. H. Rudd, A. R. Zentner, and A. V. Kravtsov, "Effects of Baryons and Dissipation on the Matter Power Spectrum," Astrophys. J. 672 (2008) 19-32, arXiv:astro-ph/0703741 [ASTRO-PH] . 
[47] R. Stanek, D. Rudd, and A. E. Evrard, "The Effect of Gas Physics on the Halo Mass Function," Mon. Not. Roy. Astron. Soc. 394 (2009) L11-L15, arXiv:0809. 2805 [astro-ph].

[48] W. Cui, S. Borgani, K. Dolag, G. Murante, and L. Tornatore, "The effects of baryons on the halo mass function," Mon. Not. Roy. Astron. Soc. 423 (July, 2012) 2279-2287, arXiv: 1111.3066.

[49] S. Bocquet, A. Saro, K. Dolag, and J. J. Mohr, "Halo mass function: Baryon impact, fitting formulae and implications for cluster cosmology," Mon. Not. Roy. Astron. Soc. 456 no. 3, (2016) 2361-2373, arXiv:1502.07357 [astro-ph.C0].

[50] T. K. Chan, D. Kereš, J. Oñorbe, P. F. Hopkins, A. L. Muratov, C. A. Faucher-Giguère, and E. Quataert, "The impact of baryonic physics on the structure of dark matter haloes: the view from the FIRE cosmological simulations," Mon. Not. Roy. Astron. Soc. 454 no. 3, (2015) 2981-3001, arXiv:1507.02282 [astro-ph.GA].

[51] G. Despali and S. Vegetti, "The impact of baryonic physics on the subhalo mass function and implications for gravitational lensing," Mon. Not. Roy. Astron. Soc. 469 no. 2, (2017) 1997-2010, arXiv:1608.06938 [astro-ph.GA].

[52] L. Hui, J. P. Ostriker, S. Tremaine, and E. Witten, "Ultralight scalars as cosmological dark matter," Phys. Rev. D95 no. 4, (2017) 043541, arXiv:1610.08297 [astro-ph.CO].

[53] J. Zhang, J.-L. Kuo, H. Liu, Y.-L. S. Tsai, K. Cheung, and M.-C. Chu, "The Importance of Quantum Pressure of Fuzzy Dark Matter on Lyman-Alpha Forest," Astrophys. J. 863 (2018) 73, arXiv:1708.04389 [astro-ph.C0]. 